\title{
El tejido de interacción escolar como potenciador de la gestión directiva en la política de inclusión ${ }^{1}$
}

\author{
John Alejandro Sepúlveda Méndez², Mónica Soto Rivas ${ }^{3}$ \\ Beatriz Ramírez Aristizabal ${ }^{4}$
}

\begin{abstract}
Resumen
El presente artículo desarrolla una propuesta integradora y de interacción, la cual partiendo de la metáfora biológica del tejido vivo llamado -tejido de interacción escolar- (TIE), muestra de manera directa la vitalidad que representa la escuela para la sociedad y la importancia de la construcción de interacciones positivas y de apertura entre sus miembros.

El artículo emerge de la categoría Clima Escolar, producto de la investigación Comprensiones y representaciones que se derivan de la incorporación de la política de inclusión desde la gestión directiva en las instituciones educativas Los Quindos y Camilo Torres del municipio de Armenia, Quindío, Colombia 5 . En este proceso investigativo se develaron una serie de factores que favorecen, o no, la implementación de las políticas públicas de inclusión, los cuales se categorizaron desde una perspectiva, tríadica: factores limitantes, concebidos como condiciones ausentes o insuficientes que afectan el colectivo o la organización, factores inhibidores impiden y suspenden el desarrollo y los factores potenciadores encargan de impulsar, dar fuerza, respaldar y favorecer.

Finalmente el artículo propone la necesidad de trascender, las estrategias de inclusión y calidad basadas en los recursos económicos, la infraestructura, el acceso, la cobertura y la permanencia de los estudiantes para construir y pensar desde la gestión directiva como fibra direccionadora tejidos interactivos escolares donde la apertura y el reconocimiento hacia
\end{abstract}

1 Recibido: 29 de julio de 2013. Aceptado: 26 de octubre de 2014.

2 John Alejandro Sepúlveda Méndez. Magister en educación desde la diversidad; Psicólogo Universidad de Manizales, Especialista en Gerencia de Servicios de Salud Universidad Cooperativa de Colombia, Máster en aspectos evolutivos, psicoeducativos, psicopatológicos y sociales de la adolescencia Universidad de Valencia, Secretaría de Educación de Armenia, Institución Educativa Los Quindos. alejo3112@yahoo.com

3 Mónica Soto Rivas. Magister en educación desde la diversidad; Trabajadora Social Universidad de Caldas, Especialista en Administración de Recursos Humanos Universidad de Manizales, Licenciada en Ética y Desarrollo Humano Universidad el Bosque. Secretaría de Educación Municipal de Armenia, Área Calidad Educativa.monicasotorivas@hotmail.com

4 Beatriz Ramírez Aristizabal. Doctorante en Ciencias sociales, Niñez y Juventud Universidad de ManizalesCinde. Magister en Educación. Docente e investigadora Universidad de Manizales. Correo electrónico: bramirez@umanizales.edu.co

5 Consideraciones, el presente artículo es el resultado de la investigación "comprensiones y representaciones que se derivan de la incorporación de la política de inclusión desde la gestión directiva", que hace parte del proyecto de investigación científico y tecnológico "Concepciones, creencias y prácticas de la Gestión Escolar", perteneciente a la línea de investigación en gestión educativa, liderada por la Dra. Beatriz Ramírez Aristizabal. 
el otro, el afecto, la capacidad de escucha, la acogida, el dialogo, el respeto mutuo, la participación, la comunicación, la construcción y mantenimiento de relaciones significativas en red y la corresponsabilidad de los actores educativos, se convierten en potenciadores para todos los procesos inherentes de la escuela, especialmente la implementación de las políticas públicas de inclusión.

Palabras claves: Clima escolar, Interacción escolar, gestión directiva, tejido escolar, políticas de inclusión, factor potenciador, actores educativos.

\section{Abstract \\ School weaving interaction as an enhancer of executive management in the politics of inclusion}

The current article develops an integrative proposal of interaction that starts from a biological metaphor of human tissue called school interactional tissue and shows in a direct way the vitality that school represents for society and the importance of the construction of positive interactions and the opening among their members.

The article emerges from school climate category as a product of comprehensions and representations investigation which is derived from the incorporation of inclusion policy from management direction in the public institutions "Los Quindos and Camilo Torres" from the municipality of Armenia, Quindío, Colombia.

In this investigative process we revealed a series of factors that favor, or not, the implementation of public policies of inclusion, which were categorized from a triadic perspective: limiting factors, conceived as absent or insufficient conditions that affect the collective or the organization, inhibitor factors that prevent and suspend the development and enhancer factors responsible for boost, strength support and encourage.

Finally the article proposes the need to transcend inclusion and quality strategies based on economic resources, infrastructure, access, coverage an permanence of students to build on and think in management as a directional fiber of school interaction networks where openness and recognition towards the other, affection, ability to listen, reception, dialogue, mutual respect, participation, communication, construction and maintenance of significant relationships in network and co-responsibility of educational actors, become enhancers for all the inherent processes of the school, especially the implementation of public inclusion policies.

Keywords: school climate, school interaction, school management, school tissue, inclusion policies, booster factor and educational agents. 


\section{Aglomeraciones} diferenciales del tejido escolar

En el presente artículo se toma como eje nodal el desarrollo de la categoría emergente "CLIMA ESCOLAR COMO FACTOR POTENCIADOR EN LA IMPLEMENTACIÓN DE LA POLITICA PUBLICA DE INCLUSION DESDE LA GESTIÓN DIRECTIVA" la cual desde una metáfora construida a partir de las características del sistema nervioso se construye una propuesta integradora, orientadora y potenciadora denominada TIE - Tejido de Interacción Escolar- se alude a la relevancia y comprensión de la interacción escolar. En la observación microscópica del tejido encontramos componentes celulares Ilamados actores educativos (funcionarios de la Secretaría de Educación Municipal de Armenia, en adelante SEM, directivos, docentes, estudiantes y padres de familia), simbólicamente se asumen los núcleos de la célula como las subjetividades (comprensiones y representaciones de los actores), la matriz externa del tejido entendida como superficie de absorción, lineamientos y sensibilidad hacen referencia a (la comunidad, las políticas públicas y el entorno sociocultural de la escuela).

Se considera que el "tejido" esta constituido por dos tipos de células, una clase de células donde todas son iguales y otro tipo de células diferentes las cuales están ordenadamente y dispuestas para cumplir mediante la interacción una función especializada y determinada llamadas microtejidos (el aula y equipos de trabajo), finalmente se identifica la fibra direccionadora y gruesa que transporta la información con sus potenciales de acción desde la matriz externa hasta las profundidades del tejido (entendida como la gestión directiva que orienta y potencia las políticas a la escuela).

\section{Matriz externa}

La comunidad, las políticas públicas y el entorno sociocultural de la escuela se consideran elementos que conforman la matriz externa, en este orden de ideas es necesario destacar actores del orden supranacional que comienzan a tener injerencia en la definición de lineamientos en la formulación de políticas públicas educativas para los países que requieren créditos de la banca internacional, entre estos actores cabe señalar los organismos internacionales de crédito, tales como el FMI o el BM, los cuales a finales de la década del 80 del siglo pasado fijaron una dirección para las políticas educativas de la región latinoamericana, algunas de estas estrategias fueron: reducir el gasto público y aumentar el recaudo fiscal para pagar los intereses de la deuda y sugieren la descentralización administrativa en educación, al igual que el fomento de escuelas privadas o que éstas sean financiadas por las comunidades (Libreros 2002, p. 23).

A estos organismos mencionados se suman entidades como la UNESCO y UNICEF, creados especialmente para impulsar el desarrollo educativo, científico y cultural de las naciones y el cuidado de la infancia. También se han realizado eventos internacionales donde los intereses por la inclusión en las políticas púbicas se evidencian, varios de ellos son el marco de acción, promulgado en la Conferencia de Salamanca (UNESCO 1994), la Convención de los Derechos del Niño de la ONU (1989) y las Reglas Estándar sobre la Igualdad de Oportunidades para Personas con Discapacidad de la ONU (1993). Dentro de estos eventos cabe resaltar La Conferencia Mundial sobre "Educación para Todos" realizada en Jomtien, Tailandia (1990), la cual marcó un nuevo inicio en la búsqueda global destinada a universalizar la educación básica y a erradicar el analfabetismo. Con esta conferencia se fijó un marco de acción para los países participantes, se asumieron compromisos y se establecieron pautas para una década de esfuerzos en la construcción de nuevas comprensiones de las interacciones y prácticas de los actores educativos. 
Por su parte a América Latina como región diversa y escenario político y social se le ha exigido construir nuevas interacciones por parte de los actores educativos como consecuencia de la implementación de políticas públicas especialmente las de inclusión, las cuales hacen carrera para buscar la igualdad de atención, acceso y permanencia en la educación, al tiempo que pretenden garantizar la expresión de la diversidad de los sujetos, frente a esto "América Latina constituye una de las regiones que presento desde la década de los setenta la distribución del ingreso más inequitativa y que en los noventa se cristaliza en dinámicas sociales atravesadas por la desigualdad social, económica y la exclusión educativa (Klisberg, 1999, p. 2).

Cabe preguntar entonces ¿sí a esas condiciones y diferencias sociales, económicas, familiares y culturales de los países latinoamericanos permiten a, la escuela realmente ser inclusiva? La respuesta no es simple, ya que se debe reconocer el esfuerzo por parte de los países en la implementación de ciertas estrategias y propuestas de los sistemas educativos para la retención de los estudiantes y culminación de ciclos educativos tales como: becas, gratuidad, restaurantes, subsidios, transporte, útiles y uniformes entre otros, al igual que la participación y el aporte de otros actores sociales como ONGs, empresarios privados, iglesias y fundaciones entre otras. Sin embargo a estas propuestas les falta identificar las nuevas interacciones sociales y las comprensiones y representaciones que surgen a partir de la implementación de las políticas públicas educativas de inclusión, lo anterior marca la diferencia con respecto a la inclusión pues a partir de la formación del tejido de interacción escolar se direccionan las políticas de inclusión.

Los gobiernos de estos países se han visto obligados a implementar políticas públicas que garanticen el ejercicio del derecho a la educación. Estas líneas de trabajo a nivel internacional y latinoamericano han vuelto su mirada al estudiante como un sujeto de derecho, sin embargo en estos países existen diferencias en los sistemas educativos, los cuales que inciden en la intención de aseguramiento del acceso, permanencia y la culminación de los niveles educativos de los niños, niñas y adolescentes.

Colombia no es ajena a la necesidad de buscar medidas y garantizar los derechos educativos de los estudiantes, evidencia de ello es la formulación de las políticas públicas de inclusión en Colombia, enmarcadas en la constitución política de 1991 y sobre un marco de derechos que se convierten en el pilar central de todas las dimensiones sociales del país apoyadas en un marco de derechos, en este sentido, la Ley 115 de 1994 General de Educación y la expedición de la Ley 60 del año 1993, donde se expiden competencias para las entidades territoriales del estado colombiano como municipios y departamentos, representan un notable avance sobre las normas que rigieron la educación colombiana a finales del siglo $X X$, ya que introdujeron un conjunto de reformas con enorme capacidad de hacer cambios importantes en la educación a mediano y a largo plazo.

Estas iniciativas legales pusieron en marcha la dirección colegiada, el gobierno escolar, los PEI y la autonomía escolar de las Instituciones Educativas resaltando la importancia de las interacciones de los actores y su participación. Siguiendo estas propuestas se presentó el documento lineamientos de política para la atención educativa a poblaciones vulnerables del Ministerio de Educación Nacional de Colombia, de ahora en adelante (MEN) El cual plantea lo siguiente:

Uno de los desafíos en materia de atención educativa es que el Estado garantice el acceso al servicio público educativo, así como la permanencia en él, tanto de los niños y niñas como de los jóvenes y adultos, sin distinciones de raza, género, ideología, religión o condición 
socioeconómica, por su naturaleza o por determinadas circunstancias. Frente a ese reto, el Plan Sectorial y la propuesta educativa llamada "La Revolución Educativa" desde un comienzo propuso diseñar e implementar procesos y acciones alrededor de tres ejes de política: mayores oportunidades para el acceso mediante el aumento de la cobertura, permanencia en condiciones de mejor calidad y puesta en marcha de las herramientas y procedimientos que aseguren la eficiencia. Lineamientos de política para la atención educativa a poblaciones vulnerables (MEN, 2005, p. 5).

La razón para que el Ministerio de Educación haya propuesto la divulgación de los lineamientos de política para la atención educativa a poblaciones vulnerables es precisamente la de entregar orientaciones y herramientas que permitan consolidar desde las mismas secretarías de educación una gestión basada en la inclusión, la equidad y la calidad del servicio educativo para poblaciones vulnerables. De esta manera el lema "Educación para todos" se convierte en una de las metas del milenio con las que Colombia se compromete, la cual busca cumplir los objetivos y finalidades de la educación para todos los ciudadanos, y mediante estos compromisos garantizar a todos los niños, niñas y jóvenes y especialmente a todos los que se encuentren en situaciones difíciles tengan acceso a la educación primaria de buena calidad mediante la implementación de estrategias que se les garantice su permanencia.

Con respecto a estos compromisos de acceso y permanencia como elementos característicos de calidad educativa, la ciudad de Armenia y su sistema educativo local reflexionan y acompañan permanente la efectividad en la implementación de las políticas públicas educativas y de las condiciones de acceso y permanencia de los estudiantes en las Instituciones Educativas de la ciudad, lo mismo que las prácticas para el aprendizaje que disminuyan la segregación de la oferta educativa y el fortalecimiento de las interacciones de los actores educativos.

Es así como desde el plan sectorial de Educación de Armenia (2012, p. 339), se hace referencia al fortalecimiento de la gestión del sector educativo; la gestión educativa se presenta como un pilar de la cultura organizacional, la cual se debe caracterizar por la capacidad de actuar en contexto, de responder al cambio, mediante el fortalecimiento de la autonomía institucional, en el marco de las políticas públicas.

En coherencia con el surgimiento de esos compromisos que presentan las Instituciones Educativas de Armenia, emerge la inquietud por explorar y conocer mediante el proceso investigativo denominado, "Comprensiones y representaciones que se derivan de la incorporación de la política pública de inclusión desde la gestión directiva en las instituciones educativas Los Quindos y Camilo Torres del municipio de Armenia, Quindío, Colombia”. Encontrar respuestas que reflejan el concepto que tienen los actores educativos (funcionarios de la SEM, directivos, docentes, estudiantes y padres de familia) sobre las políticas públicas de educación inclusiva y la comprensión que tienen de la propuesta "Educación para todos".

Dentro de los actores a los que se les interrogo sobre la política de inclusión, se encuentran algunos funcionarios de la entidad municipal, dentro de los cuales uno de los entrevistados expresa: "Pues digamos, el conocimiento que tengo es el que me da un poco la experiencia y el reconocer que... que por norma el sistema educativo está obligado a generar todas las condiciones que permitan... el, acceso de los niños al sistema educativo, y no solo el acceso, sino su promoción y su aprendizaje, y que en esa medida la escuela o el estado está en la obligación de darle a ese niño que tiene... digamos unos requerimientos especiales, una 
atención que le permita adaptarse, aprender más fácil eh... construir digamos un proyecto de vida que le permita ser... útil socialmente $y$, no tenga limitaciones y que en ese propósito la escuela debe tratarlo a él digamos... diseñando unos programas y unas estrategias que estén hechas a su particular condición. Ese es un esfuerzo que hace hacemos todos los maestros, que obviamente necesita de muchos recursos, que necesitamos más capacitación, pero en el cual yo diría que... hay un elemento muy importante que tiene que ver y yo diría que es el amor que el maestro tenga por esa tarea."

Se llega con grandes expectativas a la Secretaria de Educación, esperando encontrar un conocimiento e interpretación clara y amplia de las políticas públicas de inclusión donde el organismo orientador de las políticas públicas de educación traza estrategias y direccionamientos para las instituciones educativas. Realmente se encuentran unos conocimientos y apreciaciones muy semejantes a los escuchados en los directivos y docentes y a los propuestos por la política del Ministerio de Educación Nacional, la accesibilidad a las instituciones y la relación de la inclusión con las limitaciones, con lo especial, con el déficit, con la minoría.

También nos recuerda la necesidad de destinar recursos para direccionar e implementar las políticas de inclusión y satisfacer la necesidad de capacitación del cuerpo docente, pero también refleja el afecto del maestro hacia sus estudiantes como condición necesaria para la inclusión. En esa lógica jerárquica y microscópica de acercamiento a lo local y acudiendo a los actores educativos para comprender la permeabilidad de la matriz externa (políticas públicas) sobre ellos, se acude a los directivos docentes y se toma una muestra de las entrevistas realizadas que reflejan las comprensiones que se tienen de inclusión: Pues generales [...] cierto, generales es que ya las instituciones educativas debemos atender casi que todo tipo de población que debemos hacer todas nuestras adaptaciones curriculares de acuerdo a la necesidad de cada niño, tenemos niños que solamente trabajamos en socialización, sin embargo entender eso para el docente es muy complejo[...] sobre todo y pues para la familia también, pero la mayoría de docentes tienen el esquema de alcanzar los mismos logros con todos los estudiantes entonces... la política es de todos los niños dentro del sistema,... no señalar, no discriminar la participación de ningún niño bajo ninguna circunstancia en las instituciones educativas . ${ }^{6}$

Estas expresiones llevan a reflexionar frente al momento que algunas Instituciones Educativas presentan con respecto a las políticas públicas de inclusión y al direccionamiento y orientación que desde la gestión directiva realizan, primero que la mayoría de instituciones educativas están en un proceso incipiente de integración, vinculando a los estudiantes, pero con la única respuesta de realizar adaptaciones de tipo curricular y en algunos casos de tipo evaluativo con consideraciones especiales pero sin estrategias para la retención y la culminación exitosa de sus niveles de formación, allí se encuentra un esquema tradicional de educación homogenizante el cual genera resistencia y apreciación de sobrecarga para el docente en el aula. Sin embargo se encuentran en esta muestra de entrevista la comprensión de educación para todos donde la discriminación y la exclusión no se pueden presentar bajo ninguna justificación.

Avanzando con las células (actores educativos) integrantes del tejido Interactivo escolar se profundiza con el actor docente: [...] bueno, la educación inclusiva [...] es un pro [...] es, un producto digámoslo así, no, o es una parte del proceso que viene, que se ha estado de mucho tiempo atrás que atención a la diversidad, que el multiculturalismo, que, que la, que la inclusión, que niños con barreras, que escuela in[...] bueno, e [...]ha sido una [...]

6 Entrevista 1 
digámoslo ha sido una política gubernamental noble, tiene una filosofía noble, pero de lo dicho al hecho, como dicen pues, que pena utilizar refranes aquí, hay mucho trecho, de lo que está escrito a lo que se hace no hay nada [...] una cosa es lo que está escrito y la realidad va por otro camino lastimosamente como casi todo en nuestro país. ${ }^{7}$

Llama la atención de esta narrativa, la referencia a términos construidos y sobre los cuales se apoyan las políticas educativas para direccionar las organizaciones educativas, multiculturalismo, diversidad e inclusión, los cuales establecidos como conceptos y sustentados en un discurso teórico sufren el llamado desfase del que habla Zemelman cuando menciona que: Al no tener conciencia que se está dando un desajuste entre la teoría y la realidad que se pretende denotar, resulta que estamos inventando realidades. Situación que podemos reconocer no solamente en el ámbito de la investigación o de la docencia, de la academia en general, sino que también en otro orden de discursos, por ejemplo el discurso político (Zemelman, 2008, p. 2).

Uno de los actores más significativos, son los estudiantes, a los cuales se les interrogó ¿Qué entiende por "Educación para todos"?, cuestionamiento relevante en el sentido de indagar sobre la razón de ser de la educación, al encontrar en una de las respuestas brindada por uno de ellos: "Educación para todos, es educación para ricos, pobres [...] la [...] todas las personas que quieran estudiar ${ }^{8}$ Este tipo de expresiones muestra la comprensión de la igualdad para todos los sujetos como una propuesta de reconocimiento y oportunidades para todos, igualmente se resalta el factor motivacional del estudiante como condición para acceder y aprovechar las oportunidades. Finalmente se pregunta al padre de familia, ¿Qué entiende por "Educación para

$7 \quad$ Entrevista 8

8 Entrevista 22 todos"? Pregunta fundamental para el actor corresponsable de la formación del estudiante y responde: Ya [...] Pues que [...] no debe [...] no se debe de pronto discriminar a las personas, por [...] por eso que de pronto este no lo recibimos o de pronto una niña que de pronto que embarazada o algo, entonces vamos a dejarla de por fuera, debería ser pues [...] equitativo para todos, parejo"g

Para estos acudientes la accesibilidad y la superación de barreras como las condiciones y situaciones que presentan los estudiantes debe ser "Educación para Todos" al igual que no la discriminación de los estudiantes. Las muestras de entrevistas anteriores realizadas a los diferentes actores educativos llevaron a considerar varias barreras que afectan la permeabilidad hacia las células llamadas actores educativos, por un lado los recursos destinados a la socialización y a la divulgación de las políticas públicas de inclusión al igual que la sensibilización y capacitación para directivos y docentes, los recursos que requieren la contratación de profesionales de apoyo como psicólogos, terapeutas, trabajadores sociales entre otros, al igual que materiales diagnósticos y pedagógicos. Otra barrera que se refleja en las entrevistas es la información insuficiente por parte de directivos y docentes sobre las políticas públicas; las cuales enmarcan y direccionan la formulación en lo que respecta a los recursos y a las estrategias de reconocimiento, las políticas públicas de inclusión hacen parte del espectro bidimensional de dos extremos planteado por Fraser, la cual "considera la "redistribución" y "reconocimiento" en su referencia política; es decir, como constelaciones ideales y típicas de las reivindicaciones que se discuten en la actualidad en las esferas públicas (Fraser, 2008, p. 86).

Esto significa que no solo son políticas que buscan la reivindicación social, también la reivindicación de clase por

9 Entrevista 16 
aspectos distributivos y económicos, estas políticas públicas asumen esta bidimiensionalidad aunque aparentemente sean propuestas contrarias y excluyentes, al respecto Fraser clarifica que: [...] Los dos paradigmas asumen concepciones diferentes de injusticia. El paradigma de la redistribución se centra en injusticias que define como socioeconómicas y supone que están enraizadas en la estructura económica de la sociedad." Y "el paradigma del reconocimiento se enfrenta a injusticias que interpreta como culturales, que supone enraizadas en patrones sociales de representación, interpretación y comunicación (Fraser, 2008, p. 87).

A partir de las afectaciones que generan estas inequidades, la política de inclusión no es por sí solo la panacea, las comprensiones que se derivan de esta implementación deben ser permeadas por la propuesta del tejido de interacción escolar-TIE-, como posibilidad de encuentro con el otro.

\section{Clima escolar}

Teniendo en cuenta las definiciones positivistas, explicativas y descriptivas de clima escolar que existen y la necesidad de trascender el concepto hacia uno más comprensivo, integrador y potencializador como es el Tejido de Interacción Escolar, se hace necesario abordar algunas definiciones de clima escolar que permitan diferenciar y comprender los conceptos de clima escolar y tejido de interacción escolar.

Frente a estas razones es pertinente revisar de donde surge, cómo es comprendido el concepto de clima escolar, cuáles son los elementos constitutivos del clima escolar y cuáles autores le dan relevancia a uno u otro elemento. Al respecto se identifica que el origen del concepto de clima escolar, se puede situar en la psicología social y en el enfoque de sistemas. Con respecto a las raíces del clima escolar
Tagiuri y Litwin, (1968) y Schneider (1975), plantean que el desarrollo del concepto de clima escolar tiene sus orígenes en el concepto de "clima organizacional", el cual es el resultado del estudio de las organizaciones y del campo laboral los cuales se consolidan a finales de la década de 1960, en este origen de concepto se refleja el aporte de la psicología social para explicar el comportamiento de las personas en las organizaciones.

El clima escolar entonces ha partido de una propuesta descriptiva y explicativa del mundo de las organizaciones, configurándose una gama amplia de conceptos, unos centrados en la relevancia de las estructuras y los procesos, algunos fijando la atención en la cultura organizacional, los estilos de dirección y los procesos comunicativos y otros centrados en las percepciones de los integrantes de la organización. Isidora y V. mencionan que: En las últimas décadas se ha desarrollado un creciente interés por el estudio del clima organizacional, asociado a su inclusión como indicador de la calidad de la gestión de las organizaciones y como uno de los elementos básicos para potenciar procesos de mejoramiento al interior de éstas. Estos autores al estudiar el desarrollo del concepto, reconocen 22 definiciones, dando cuenta de las dificultades y discrepancias para precisar el significado de este constructo. Al analizarlas, reconocen cómo se enfatizan las estructuras v/s los procesos de la organización, revelando en distintos grados su carácter objetivo v/s subjetivo. (Mena y Valdés, 2008, 2)

Precisamente esos énfasis han polarizado las definiciones de clima organizacional identificándolas como variables independientes y dependientes desde la perspectiva positivista de las organizaciones. Esta tendencia ha trascendido al medio educativo hasta el punto de encontrar definiciones de clima escolar en estas polaridades, al respecto se pueden apreciar algunas definiciones como las siguientes: Arón y Milicic (1999) lo definen 
como» la percepción que los miembros de la institución escolar tienen respecto del ambiente en el cual desarrollan sus actividades habituales». Estas percepciones entonces se apoyan en la experiencia que los actores tienen en su lugar de trabajo. Por otra parte encontramos ejemplo de definiciones donde se reconoce la propuesta de variadas dimensiones que componen este constructo.

Entre ellas prevalecen las relaciones que se establecen entre los distintos actores escolares, los elementos relativos al funcionamiento de la organización y condiciones físicas del ambiente (Becerra, 2007; Stevens, 2007, Debarbieux, 1996, Justiniano, 1984). Frente a estos componentes es interesante reconocer que el clima escolar no necesariamente homogeniza o uniforma a toda la institución. El estudio del clima escolar puede estar centrado en procesos que ocurren al interior de la vida escolar, frente a estos componentes cabe la posibilidad de asociar y relacionar al clima escolar con otros factores y condiciones del mundo escolar lo cual genera una multiplicidad y variada posibilidad de definiciones.

En estas posibles relaciones se encuentra el clima escolar asociado a la calidad educativa, dentro de ellas cabe mencionar a Teodoro (2006), el cual plantea lo siguiente:

1. Encontramos el liderazgo desde una propuesta democrática que debe partir del equipo directivo, 2. La corresponsabilidad de todos los actores educativos institucionales en la transversalidad e interacción permanente, recuerden que sin interacción es imposible generar tejidos escolares positivos y procesos de inclusión, 3. La comunicación efectiva y dialógica dentro de la institución, expandiendo la capacidad de escucha de los directivos y docentes, en este caso cabe señalar la importancia de promover respuestas asertivas en la comunicación, 4. Las relaciones respetuosas, acogedoras y cálidas dentro del aula, aquí se resalta la importancia de considerar y valorar los microtejidos escolares, 5. Las relaciones respetuosas entre los miembros de la institución en los espacios escolares fuera del aula; $y$ 6. Las relaciones participativas de la institución con el entorno escolar, es decir esas relaciones que se construyen con la comunidad, iglesia, entidades públicas y privadas y universidades entre otras. (Teodoro, 2006, p. 123).

En concordancia con las características del clima escolar descritas por Pérez (2006), el Ministerio de Educación Nacional de Colombia (2008), propone una definición de clima escolar como es "Generar un ambiente sano y agradable que propicie el desarrollo de los estudiantes, así como los aprendizajes y la convivencia entre todos los integrantes de la institución." (MEN, Guía 34, 2008, 29). Además de esta definición el MEN plantea unos procesos integrales y necesarios para el clima escolar como son: la pertenencia y participación, ambiente físico, inducción a los nuevos estudiantes, motivación hacia el aprendizaje, el manual de convivencia, la realización de actividades extracurriculares, los programas en cada Institución de bienestar de los alumnos, y el manejo eficiente y oportuno de conflictos y casos difíciles. Estos componentes del clima escolar propuestos por el MEN de Colombia resultarían impactantes para generar un clima escolar potenciador si se llevaran a cabo de manera articulada y direccionada no solo por la gestión directiva, también por las otras gestiones y proyectos pedagógicos Institucionales.

De acuerdo a esta guía 34 del Ministerio de Educación Nacional se ha convertido actualmente en la herramienta más utilizada por directivos y docentes para realizar mediciones de las gestiones institucionales., ya que permite identificar fortalezas y debilidades de las instituciones, en estos 
procesos la medición del clima escolar es tangencial y poco prioritaria, pues se puntualiza de manera significativa en los resultados de la gestión académica, dando respuesta a exigencias de las políticas nacionales de calidad educativa, sería un gran avance evaluar las percepciones de las relaciones que se gestan al interior de la vida escolar y de igual manera el conjunto de elementos procesuales y característicos que hacen parte del clima escolar.

Desde estas intencionalidades de medición del clima escolar, este nunca será neutro, siempre impactara, ya sea actuando como favorecedor u obstaculizador del logro de los propósitos institucionales. En términos generales, los climas escolares potenciadores o favorecedores de la organización son aquellos en los cuales:

Se facilita el aprendizaje de todos quienes lo integran; los miembros del sistema se sienten agradados y tienen la posibilidad de desarrollarse como personas, lo que se traduce en una sensación de bienestar general, sensación de confianza en las propias habilidades, creencia de la relevancia de lo que se aprende o en la forma en que se enseña, identificación con la institución, interacción positiva entre pares y con los demás actores. Los estudiantes se sienten protegidos, acompañados, seguros y queridos". (Arón y Milicic, 1999, p. 67).

Complementario a estas características de clima escolar se hace necesario retomar una definición con una aproximación más integradora propuesta por Rodríguez, el cual plantea que éste corresponde a las "percepciones compartidas por los miembros de una organización respecto al trabajo, al ambiente físico en que éste se da, las relaciones interpersonales que tienen lugar en torno a él y las diversas regulaciones formales que afectan a dicho trabajo." (Rodríguez, 2004, 147). Con respecto al concepto de las percepciones, estas son concebidas como "el conjunto de interpretaciones y significados que construye el sujeto, al hacer subjetivo un mundo objetivo o real" (Sepúlveda, 2002, p. 1). Estas percepciones entonces generan disposición y motivación para trabajar en equipo y en beneficio de la organización.

Este es un factor que incide en la calidad educativa y que se relaciona con la atención a los padres y el tiempo que se le dedica a ellos, en estos se resalta la expresión de muchos docentes frente a esta actividad refiriéndose que no les alcanza el tiempo, debido a la complejidad de las problemáticas que expresan los acudientes cuando recurren a ellos y en ocasiones están referidas a lo económico y a las difíciles relaciones con sus hijos, situación que les ha generado a los directivos docentes replantear y priorizar estas actividades ampliando, liderando y programando los espacios institucionales para que esta atención produzca acogida y buen ambiente en la Institución Educativa.

Por su parte la investigación Comprensiones y representaciones que se derivan de la incorporación de la política de inclusión desde la gestión directiva en las instituciones educativas Los Quindos y Camilo Torres del municipio de Armenia, Quindío, Colombia, gestaron categorías alternas derivadas desde los factores inhibidores y limitantes, definidas respectivamente así: La no comprensión del concepto de una escuela para todos como inhibidor en la aplicación de la política pública de inclusión, la ausencia de la gestión directiva como limitante en la atención a la diversidad en las instituciones educativas, las cuales posibilitaron identificar las diferentes percepciones que tiene la población objeto sobre la incorporación por parte de los directivos docentes de la política pública de inclusión y se resalta de manera puntual el clima escolar como factor potenciador de la incorporación de la política pública de inclusión. 
Esta categoría emergente se vio reflejada en las respuestas de los actores entrevistados referidas a las relaciones reinantes en la Institución, se resalta las realizadas a un grupo de estudiantes, para lo cual se referencian como muestras las respuestas de los entrevistados 23 y 24 , a los cuales se les pregunta por las relaciones establecidas dentro de la institución, estos afirman que: "son muy... como unidos, siempre los docentes escuchan a los alumnos y es una relación buena, no hay tanto conflicto." (Entrevista 23.), otro actor responde "la convivencia siempre es buena", "yo he visto que todas las personas que llegan acá se las trata muy bien." Esta percepción no dista del grupo de padres de familia, ya que las entrevistas realizadas a estos develan la aprobación de las relaciones que se dan en la comunidad educativa, destacando la buena convivencia entre estudiantes, docentes, directivos y padres de familia que en conjunto son afectados positivamente por la interacción social dentro de la institución.

Refrendando esta percepción se evidencia en las entrevistas a padres de familia los entrevistados 19 y 21 , quienes expresan los siguientes aportes: "Pues hasta donde yo sé, son muy buenas Sí, porque ellos tienen mucha conexión, sí, comparten mucho", "Pues a mi concepto es muy buena, es excelente". De igual forma, varios docentes entrevistados califican el clima escolar de las dos instituciones como armonioso, ya que las relaciones que se dan dentro de las mismas están basadas en el respeto, el trato humano y la comunicación, esto se hace patente cuando describen de la siguiente manera las relaciones entretejidas al interior de sus instituciones: "en general bien, son relaciones mediadas por, por mucho diálogo, por diálogo y respeto", "Esta comunidad particularmente, es una comunidad de paz, de los padres se caracterizan por ser muy buenos, muy, de mucha aceptación del maestro", "hay buena conexión entre los directivos, los padres de familia y los profesores, hay diálogo siempre", "se vive un ambiente entre los estudiantes de respeto, de cordialidad", "acá el trato es muy humano".

Por último, los directivos docentes entrevistados al momento de describir la convivencia en su institución refuerzan la sensación de agrado o satisfacción al emitir comentarios como: "Yo creo que son muy positivas, sin dejar de decir que hay diferencias que es lógico que las halla entre tantas personas", "cuando llega el muchacho se le da un buen trato", "hay una cosa que se llama actitud frente al frente al niño, eh... ya uno ve que los maestros tratan mejor a los niños".

Esta manifiesta satisfacción por el clima escolar vivido por los diferentes grupos poblacionales en las instituciones estudiadas - Camilo Torres y Los Quindos-, en ultimas se traduce en reconocimiento institucional, pues son repetitivas las características de calidad que los entrevistados le asignan a sus establecimientos al momento de referirse a ellos, dotándolos de esta manera de un renombre construido a partir de la comparación y la experiencia.

\section{Del clima escolar al Tejido de Interacción Escolar (TIE)}

Teniendo en cuenta los diferentes conceptos de clima escolar, sus tendencias bidimensionales de subjetividad y objetividad, la perspectiva fragmentada, instrumental y de producto que plantea cada definición, se establece la necesidad de construir una definición y perspectiva integradora, comprensiva e interactiva de clima escolar a la cual se le denomina Tejido de Interacción Escolar, a continuación se describen las características de esta propuesta diferenciadora entre clima y tejido de interacción escolar: 
DEL CLIMA ESCOLAR AL TEJIDO DE INTERACCIÓN ESCOLAR (TIE)

\begin{tabular}{|l|l|}
\hline \multicolumn{1}{|c|}{ CLIMA ESCOLAR } & \multicolumn{1}{c|}{ TEJIDO DE INTERACION ESCOLAR } \\
\hline Postura Explicativa y Descriptiva & Postura Comprensiva e Integradora \\
\hline $\begin{array}{l}\text { Énfasis en las relaciones basados en los resultados del } \\
\text { aprendizaje }\end{array}$ & $\begin{array}{l}\text { Énfasis en la Interacción del actor educativo con el otro y } \\
\text { con los otros }\end{array}$ \\
\hline $\begin{array}{l}\text { Relevancia de estructuras y procesos como: cultura } \\
\text { organizacional, estilos de dirección, procesos comunicativos }\end{array}$ & $\begin{array}{l}\text { Relevancia en la configuración de redes conectivas de sentido } \\
\text { entre los sujetos. }\end{array}$ \\
\hline Construcción y mantenimiento de relaciones funcionales. & Construcción y Mantenimiento de relaciones significativas. \\
\hline Relaciones Lineales & Relaciones en Red \\
\hline Medio físico, social e interacciones & $\begin{array}{l}\text { Representaciones y comprensiones que construyen los actores } \\
\text { educativos frente a lo que sucede }\end{array}$ \\
\hline $\begin{array}{l}\text { Mirada técnica, instrumental, de medición, control, predicción } \\
\text { y de observación e intereses de resultados }\end{array}$ & $\begin{array}{l}\text { Mirada en los procesos educativos y en la participación para } \\
\text { la construcción del presente histórico }\end{array}$ \\
\hline La escuela enseña & La escuela es un escenario para que aprendamos \\
\hline Con respecto a la diversidad expresión de tolerancia por el otro. & $\begin{array}{l}\text { Con respecto a la diversidad Expresión de actos de conciencia } \\
\text { cotidiana de respeto por el otro. }\end{array}$ \\
\hline
\end{tabular}

Construcción propia de los autores

El tejido de interacción escolar (TIE) como cualquier tipo de tejido vivo cumple una función y presenta algunas condiciones especiales que dinamizan y garantizan la adecuada organización, participación y conectividad de las células constitutivas (actores educativos). A estas condiciones se suma la calidad y el estilo de sus interacciones, en este sentido, en los últimos años existe cierta preocupación por intervenir en la calidad de las interacciones de los actores del sistema educativo, como condicionante de una gestión de calidad hacia la consecución de los deseados resultados de aprendizaje (Murillo y Becerra, 2009, 378). Así mismo la colaboración entre los miembros de la Institución Educativa, el trabajo en equipo, la coordinación de tareas, el respeto y la comunicación entre otros son condiciones necesarias para lograr un equilibrio importante de adaptación y ajuste de los actores educativos.

Este TIE participa y es primordial para la función vital de formación de la escuela. Recordemos que la escuela es vida y el eje de desarrollo de una sociedad y de un país, por ello es necesario tomar el microscopio y empezar a identificar en el tejido las células (actores educativos), a su núcleo (Comprensiones y
Representaciones) y sus moléculas (la participación, pertenencia de los actores y su convivencia) se generan cuando se conectan con la matriz externa (políticas públicas de inclusión) a partir de una fibra y estructura conductora llamada la gestión directiva.

Hacer mención del Tejido de Interacción Escolar remite a definiciones que se aproximan al propósito de construcción de este concepto integrador, entre ellos encontramos a Cere haciendo referencia al clima escolar, el cual afirma que:

"es el conjunto de características psicosociales de un centro educativo, determinados por aquellos factores 0 elementos estructurales, personales y funcionales de la institución que, integrados en un proceso dinámico específico, confieren un peculiar estilo a dicho centro, condicionante, a la vez de los distintos procesos educativos" ( Cere, 1993, 30).

Esta definición permite comprender la importancia de aspectos individuales y sociales, donde la estructura de la Institución Educativa, el estilo de dirección, las percepciones de los sujetos y las interacciones de ellos se integran de una manera dinámica para producir unas 
fibras integradoras y potenciadoras desde la matriz externa hasta las profundidades de los tejidos, es así como se consolida y confiere una identidad, una personalidad institucional y una diferenciación con otros tejidos escolares.

Con respecto a lo anterior, surge la pregunta ¿Por qué hablar de interacción en el tejido escolar? Para dar respuesta, es necesario realizar una serie de reflexiones: Primero, hay que señalar que la escuela moderna entendida como Institución u Organización favorece la interacción del actor educativo con el otro y con los otros, promoviendo la creación de redes conectivas entre los sujetos los cuales le dan sentido a la cotidiana vida escolar. Estas redes conectivas conducen a los miembros de la Institución a construir y mantener relaciones significativas que determinan formas particulares de ser, actuar, pensar y sentir.

Estas redes sufren las tensiones, angustias, impotencias e incertidumbres que producen los cambios exigidos, reformas, rupturas de paradigmas y nuevas políticas diseñadas para la escuela de hoy. Generando nuevos estilos de relación entre las células del tejido (actores educativos) y a su vez presentando afectaciones en la matriz externa en sus espacios familiar, comunitario, laboral y ciudadano.

Segundo, es necesario mencionar y tener en cuenta que la interacción de los actores educativos al funcionar como una intrincada red de relaciones y de interacciones entre los sujetos conecta a las familias, las comunidades y a los actores con la comunidad y con las instituciones educativas, las cuales generan un proceso de retroalimentación recíproca entre familia, institución educativa y sociedad. De esta forma la interacción como red nunca permanece sola al contrario se comunica con un entorno o medio ambiente escolar, al respecto Cano y Lledó afirman que:

El medio escolar. [...] jamás es neutro, articula un conjunto de infor- maciones propias que, probabilísticamente, inhibe o desencadena, facilita u obstaculiza, pautas de conducta ya latente, modos de pensamiento, formas de sentir, etc. Así concebido el entorno constituye un medio de comunicación no verbal que interacciona con el individuo, que habla por él y con él [...] (Cano y Lledó, 1990, p. 12).

Teniendo en cuenta este entorno donde se presentan las interacciones y las afectaciones que se producen en los actores educativos se ve la necesidad de trascender los conceptos de clima escolar, ambiente escolar, medio escolar o entorno escolar y utilizar el concepto de tejido de interacción escolar- TIE-, el cual trasciende el positivismo y la funcionalidad de las relaciones de los miembros, el espacio físico y los recursos para asumir una comprensión del mundo escolar como escenario vital de la sociedad de , las interacciones que se producen en dicho medio y de la construcción de las representaciones y comprensiones que los actores educativos establecen frente a los cambios.

EI TIE da vida e identidad a las instituciones, por lo tanto es vital que éste refleje la fibra gruesa del direccionamiento estratégico de la escuela, el TIE que vive la institución educativa permite conocer la dinámica institucional y es un sello de la misma., ya que es particular y emerge del entramado o tejido de carácter único dado por las características propias del contexto. Contexto que en ultimas potenciará, limitara o inhibirá los diferentes procesos institucionales. De esta manera se espera que en un tejido de interacción escolar (TIE) potenciador e integrador, los resultados en términos de procesos formativos sean los deseados.

Razón por la cual es fundamental que los miembros de la comunidad educativa perciban un TIE de bienestar, de confian$\mathrm{za}$, de afecto y de exigencia, entendido desde la perspectiva de Pérez, (2006) 
como la exigencia con respeto... no es condescendencia, es exigencia con respeto, calidez y cariño, ya que con ello se contribuye a garantizar no solo el acceso a la vida escolar, sino también la permanencia en la misma y la culminación exitosa de los ciclos de formación.

A partir de lo anterior y de acuerdo a la investigación realizada se puede decir que los directivos docentes de ambas instituciones describen el clima escolar de sus instituciones como de calidad, pues tal y como lo menciona Pérez este se caracteriza por:

La comunicación efectiva y dialógica dentro de la institución, expandiendo la capacidad de escucha de los directivos y docentes; IV) las relaciones respetuosas, acogedoras y cálidas dentro del aula; V) las relaciones respetuosas entre los miembros de la institución en los espacios escolares fuera del aula. (Pérez, 2006, p. 3)

También se puede afirmar que las instituciones educativas Camilo Torres y Los Quindos cumplen con las condiciones para un (TIE) Tejido de Interacción Escolar potenciador, de acuerdo a la definición de tejido propuesta en el artículo. Esto se refleja en las respuestas de los entrevistados de los diferentes grupos poblacionales, los cuales realizan manifestaciones que dejan ver la aprobación y el agrado por el TIE. Esta sensación de bienestar vivida dentro de las instituciones, es el resultado de un entretejido de cordialidad, respeto, interés y compromiso que caracterizan las relaciones establecidas entre los miembros de la comunidad educativa.

Frente a la cordialidad y el buen trato surge una pregunta extendida a las Instituciones Educativas y al sistema educativo en especial. ¿Porque es importante el (TIE) para la educación?

La respuesta surge de analizar que en ambas instituciones para los directivos la comunicación es un aspecto muy importante, en las relaciones entre la comunidad educativa. Encontramos que solamente un directivo de la institución Camilo Torres se refiere al "buen trato" como un elemento importante en la transformación de los procesos institucionales, en comparación con la institución Los Quindos donde los directivos frente a preguntas relacionadas con las características que debe tener una institución y un docente que atiende la diversidad, centran sus respuestas en el clima institucional, en el buen trato y en el respeto. Por último, los directivos docentes entrevistados al momento de describir la convivencia en su institución refuerzan la sensación de agrado o satisfacción al emitir comentarios como: "Yo creo que son muy positivas, sin dejar de decir que hay diferencias que es lógico que las halla entre tantas personas", "cuando llega el muchacho se le da un buen trato", "hay una cosa que se llama actitud frente al frente al niño, eh... ya uno ve que los maestros tratan mejor a los niños".

Es así como emerge el Tejido de Interacción Escolar (TIE), el cual se presenta y convierte en un factor potenciador que hace parte de los procesos académicos de los estudiantes, en su adaptabilidad y en un ambiente favorable de los diferentes actores y en un espacio que contribuye al desarrollo de las labores de los maestros. Este tejido de interacción trasciende el aspecto físico de las instituciones, la infraestructura y los recursos para el aprendizaje con los que cuenta. Este ha sido un indicador poco utilizado y tenido en cuenta a la hora de hablar de calidad educativa, ya que el interés ha sido cobertura, eficiencia, accesibilidad y retención, centrando el interés en los resultados de los estudiantes en las pruebas externas e internas con una mirada técnica, instrumental, de medición, control, predicción y de observación e interés de resultados pero no de los procesos educativos, ni de lo que sucede en los procesos para producir ciertos resultados. 


\section{Microtejido de interacción escolar}

Al explorar el TIE encontramos unas células (actores sociales) que forman un Microtejido escolar (Aula y Equipos de trabajo), este Microtejido se observa a partir de los núcleos perceptuales (comprensiones y representaciones) y generan unas interacciones particulares que pueden beneficiar u obstaculizar las gestiones o procesos de las Instituciones Educativas, al respecto Arón y Milicic (1999) plantean que los microclimas en la escuela pueden ser protectores para las personas cuando son microclimas positivos y se ven rodeados de climas escolares negativos, un ejemplo de este tipo son los microtejidos de aula positivos que se presentan ante Tejidos escolares negativos y conflictivos, en estos los integrantes del aula junto con el director de grupo construyen interacciones protegen y amortiguan el efecto adverso que puede producir el tejido escolar negativo.

Los microtejidos entonces se pueden definir como el conjunto de percepciones de los actores referentes a distintos subsistemas de la organización educativa. EI tejido de interacción escolar se compone de variados microtejidos, que pueden ser protectores u obstaculizadores del desarrollo institucional, independientemente del tejido escolar característico. Los microtejidos pueden ir desde la conformación de pequeños grupos al interior de un aula hasta un grupo completo como equipos de áreas, sala de profesores, grupos musicales, grupos de teatro, clubes deportivos entre otros y pueden ir desde grupos muy reducidos hasta grupos que abarquen la comunidad como las escuelas de padres.

Es así como estos Micro tejidos se constituye en factores potenciadores o inhibidores, dentro de ellos encontramos a los equipos directivos los cuales a partir de la fibra direccionadora y orientadora generan procesos de planeación, ejecu- ción y evaluación para el funcionamiento de las instituciones educativas. Se puede afirmar que el Microtejido escolar no se queda encerrado en el aula de clase o solo en lo institucional afectando única y exclusivamente a estudiantes, docentes y directivos docentes; éste trasciende y como lo menciona Pérez "El clima escolar apropiado impacta también, en forma indirecta, a la familia y a la sociedad a través de la interacción de los miembros de la institución en sus espacios de convivencia extramurales" (Pérez, 2006, p. 5).

Los impactos de los microtejidos en el tejido de interacción escolar demuestran la importancia de conformar y liderar equipos de trabajo en las instituciones educativas lo mismo que fortalecer de manera positiva las interacciones de sus miembros mediante la participación, la concertación y el reconocimiento.

\section{La fibra direccionadora y orientadora de la gestión directiva}

Al inicio del artículo se hizo referencia a una importante fibra conductora llamada gestión directiva, la cual es una línea gruesa y extensa que se extiende a lo largo del tejido interactivo escolar, esta fibra lidera, direcciona y orienta las políticas públicas recibidas desde la matriz externa y las conduce a otras fibras llamadas gestión académica, comunitaria, administrativa y financiera. Esta fibra debe liderar todos los procesos de acuerdo a la premisa " $\mathrm{La}$ Gestión Directiva se refiere a la manera como el establecimiento educativo es orientado" (MEN, 2008).

La gestión directiva, de acuerdo a los lineamientos del Ministerio de Educación nacional, incluye los procesos: "direccionamiento estratégico y horizonte institucional, la cultura institucional, el clima escolar, la gestión estratégica y el gobierno escolar, además de las relaciones con el entorno". MEN. (2008). La gestión directi- 
va entonces permite organizar, ejecutar y evaluar el funcionamiento general de las instituciones educativas, encontrando en cada una de esas etapas las oportunidades de mejoramiento de la Institución. Es muy importante que a través del equipo directivo se oriente la construcción y la definición del horizonte institucional, se direccione la posibilidad de una visión compartida, se establezcan los procesos comunicativos con sentido y se acompañen y consoliden equipos de trabajo que actúen en forma de red. Esto necesita medidas de orden pedagógico y organizativo, donde los aportes teóricos y prácticos fortalecen la gestión y el liderazgo.

Al respecto (Sacristán, 1991), mencionan que la gestión directiva se debe comprender como acción educativa donde los sujetos tienen papeles fundamentales, dentro de estos papeles la acción se muestra como unidad de análisis; donde la comprensión de lo que acontece en el mundo educativo tiene que ver con los agentes que le dan vida con sus acciones y con sus interacciones. Lo anterior resalta el valor de las acciones y la comprensión de esas acciones, de los sujetos que las realizan y las relaciones que se establecen aspectos fundamentales para entender la educación como proceso social y la posibilidad de instaurar los potenciales cambios que se pueden gestar desde los equipos directivos institucionales, los cuales se deben consolidar y fortalecer para definir, potenciar y posibilitar la incorporación de las políticas públicas de inclusión.

En este sentido es coherente la apreciación de Cassasus, el cual afirma que actualmente:

La gestión debe responder no tanto con las funciones y procesos racionalmente determinados, es necesario abordar las necesidades de los sujetos involucrados en el proceso, tales como el compromiso, y la satisfacción en el trabajo. Es decir temas micro, tales como liderazgo, satisfacción, calidad de las relaciones interpersonales, la comunicación, el clima, que son los temas sobre los cuales se basan las capacidades de cambio y de adaptación a los cambios." (Cassasus, 2000, p. 36).

En la fibra direccionadora de la gestión el liderazgo se constituye en una característica con presencia significativa, donde los directivos la pueden convertir en una posibilidad estratégica en la acción y a través de la interacción con los demás actores educativos, logrando un resultado favorable mediante la articulación entre la visión, la autoridad y el saber.

En este orden de ideas, el directivo docente demuestra su desempeño y el cumplimiento de sus funciones en la medida en que logren soluciones acertadas, concertadas, equitativas Al igual que la construcción de escenarios probables y deseables, seleccionando las mejores estrategias y reduciendo al máximo la incertidumbre. El liderazgo del directivo debe trascender y reflejarse en el mejoramiento de las interacciones de los miembros de la comunidad educativa y en la generación de cambios profundos y estables.

Estos cambios que requieren los directivos docentes de acuerdo a las nuevas demandas de la gestión directiva, ponen de manifiesto una competencia vital que debe ser desarrollada en ese tejido escolar y es un mayor liderazgo, con el fin de que ejerzan una autoridad más horizontal, promuevan mayor participación en la toma de decisiones, desarrollen nuevas competencias en los actores educativos, nuevas formas de interacción entre sus miembros, interacciones alternativas entre la organización y con otras organizaciones.

En este sentido es importante la apropiación de esa matriz externa de políticas de inclusión, la información suficiente y clara de la inclusión que le permita apropiarse y empoderarse del discurso y la práctica y así poder direccionar y orientar el transito que deben asumir las organizaciones educativas frente a la inclusión. 
Pero este empoderamiento requiere asumir y profundizar en las subjetividades de los directivos, los cuales mediante las comprensiones de los desafíos y tensiones que producen los cambios y el conocimiento claro del tipo de representaciones que ellos construyen con las políticas y exigencias del medio externo e interno de las Instituciones, pueden entonces agenciar procesos de inclusión claros y efectivos, algo así como si no evalúo mi subjetividad, es difícil comprender y afectar las interacciones de los demás actores educativos.

Se trataría entonces de hacer una ruptura paradigmática frente a la gestión directiva clásica, tradicional o cotidiana que se realiza en las instituciones educativas, la cual ha sido instrumental, técnica y de mediciones transferidas por las ciencias de la administración, Es necesario entonces replantear la gestión actual, bajo la perspectiva de un nuevo orden y propuesta de organización educativa como lo es la inclusión, precisamente la educación inclusiva requiere una toma de conciencia cotidiana sobre el respeto al otro y a los otros, un pensamiento crítico a la escuela y a las formas homogéneas y estilos estandarizados de atender a los estudiantes.

Frente a esta ruptura la inclusión puede ser entendida como un proceso de transformación de las comprensiones de los diferentes actores educativos frente a la garantía y la participación de todos los niños, niñas y adolescentes., pero para lograr lo anterior se necesitan cambios estructurales en la organización, planeación, en los estilos de hacer las prácticas pedagógicas, en la forma de evaluar y en la manera de comprender las barreras y potencialidades de los estudiantes para alcanzar el éxito escolar.

Precisamente frente a la inclusión y los beneficios de atender a la población diversa, reconocerla y al tiempo construir comunidades educativas que compartan fines comunes, se requiere gestionar a través de lo diverso y lo común. Estas características de lo diverso y común son propias de la escuela de hoy y no se puede gestionar si no es a través de lo que propone Agudelo.

Todo esto sólo será posible si la institución educativa orienta su gestión desde una acción política transformadora de imaginarios, representaciones y actuaciones de los diversos agentes que a ella confluyen, una gestión crítica y con capacidad de emanciparse de los modelos foráneos allegados de la reingeniería, la calidad en la producción industrial y el afán estandarizador del mercado." (Agudelo, 2012, p 49).

En esta nueva propuesta de organización educativa que representa, atiende y actúa sobre lo diverso, se debe resaltar la identificación de lo diverso no solo en el estudiante, también en sus familias, en los directivos y en los docentes, sin embargo deben existir unas comprensiones y actuaciones comunes para la interacción de sus miembros que muchas veces es ignorada, la lectura del otro, esa lectura que es olvidada o que simplemente no hay tiempo o no queda tiempo como consecuencia de una gestión instrumentalizada. Hay que recordar que la gestión necesita reflexión e interacción, pero también reconocimiento y contemplación con el otro. La gestión directiva debe trascender la perspectiva instrumental, caracterizada en las Instituciones educativas por el manejo eficiente y transparente de los recursos económicos, propios de una política y filosofía racional del gasto público, armonizada y complementada por una amplia concepción de la pedagogía al servicio de las comunidades diversas a partir de principios de equidad en cuanto al crecimiento y desarrollo de la institución a través del aprovechamiento de las potencialidades y oportunidades de su talento humano.

Resulta fundamental entonces que los directivos docentes fortalezcan competencias esenciales en la educación y no 
solo desde la administración, Cassasus menciona que. "es necesario desarrollar la dimensión pedagógica en la gestión educativa. Es necesario repensar la gestión educativa para que en ella la gestión no se limite a la administración de los recursos sino abarque también el liderazgo pedagógico." (Cassasus, 2000).

\section{El tejido de interacción} escolar potencia la gestión directiva en la incorporación de las políticas públicas de inclusión

Frente a la potencialidad que existe en el tejido de Interacción Escolar, se hace necesario diferenciar la potencialidad de acción y la de inhibición que existe en el tejido y las posibilidades que este tejido muestra para direccionar la matriz externa de las políticas.

Desde esta mirada diádica, se resalta el concepto de factor Potenciador, como el encargado de impulsar, fortalecer, respaldar y favorecer el desarrollo de la inclusión en la escuela y permear todas las gestiones impactando en resultados de mejor convivencia, aumento de las interacciones, mayores perspectivas de mundo escolar y mejores desempeños académicos.

Mediante el fortalecimiento del TIE se favorece el intercambio dialógico de los diferentes actores, se construyen las verdades del contexto, se comprenden las dinámicas complejas del mundo escolar y se identifican las prácticas habituales que bloquean el cambio transformador que el TIE pretende, frente a esto se afirma que, "un buen clima social escolar, unido a buenos resultados académicos, se pueden considerar indicadores necesarios y ninguno por sí solo suficientes, para demostrar que una escuela es un buen contexto para proveer el tipo de educación que se requiere para el desarrollo de los países del siglo XXI. (Mena y Valdés, 2008, p. 26)
En consecuencia, podría afirmarse que la interacción escolar se convierte en un potencial, que bien aprovechado conduce a la comprensión y a la acción, en ese sentido desde la gestión directiva como fibra conductora del tejido, se fortalece la comunicación, el estilo de liderazgo, el reconocimiento, los espacios para los encuentros de los actores y la participación para favorecer la implementación de las políticas públicas de educación inclusiva, pues según, Duarte (2005) las interacciones escolares y el papel de la comunicación son fundamentales para la superación de la concepción instrumental de la escuela moderna.

En este orden de ideas, es importante resaltar la idea de Murillo y Becerra (2009) quienes afirman que fibras como los problemas de comunicación, el manejo del poder, el estilo de dirección autoritario, la falta de tiempo para escuchar al otro y la sobrecarga de tareas, son fibras inhibidoras del tejido que afectan considerablemente la interacción escolar. Estas fibras inhibidoras del tejido, que afectan la interacción escolar y congruentes con el estudio de (Murillo y Becerra, 2009), se encuentran fibras como los problemas de comunicación, el manejo del poder, el estilo de dirección autoritario, la falta de tiempo para escuchar al otro y la sobrecarga de tareas.

A partir de estas reflexiones se puede concluir que es fundamental construir y proponer desde la gestión directiva tejidos interactivos escolares que posibiliten la apertura y el reconocimiento hacia el otro, el afecto, la capacidad de escucha, la acogida, el dialogo, el respeto mutuo, la participación y la comunicación entre sus miembros.

A la gestión directiva también se le exige el direccionamiento de una educación con calidad, basada en cobertura, calidad, eficiencia y evaluación permanente, todo esto como condiciones necesarias para responder a las exigencias de la sociedad y a la realidad actual de las políticas 
públicas, especialmente las de inclusión. Si bien es cierto es necesario asumir el aprendizaje de los estudiantes, la cobertura y el acceso desde una perspectiva técnica, estas condiciones son insuficientes para garantizar la permanencia, la motivación y los resultados favorables del aprendizaje de los estudiantes.

El tejido de interacción escolar se constituye en un proceso de construcción permanente que comprende la interacción de los sujetos la construcción de redes de sentido, las características de la Institución Educativa y las percepciones que construyen y poseen los integrantes de las entidades educativas. Este proceso llamado tejido de interacción escolar cobra importancia al considerarse parte vital y esencial de la calidad en la educación.

El Tejido de Interacción Escolar favorece la implementación de las políticas educativas públicas de inclusión al considerar a dichas políticas como un valor institucional, desde una comprensión diferente de escuela y a través de la construcción de perspectivas diferentes de los directivos y docentes que inviten a la revisión de las prácticas educativas y a los estilos de comprender, valorar y atender la diferencia.

\section{Bibliografía}

\section{Fuentes}

Agudelo, Alexandra. (2012). Aspectos Fundamentales de la Gestión Escolar. Maestría en Educación desde la Diversidad. Facultad de Ciencias Sociales y Humanas. Manizales: Universidad de Manizales, CEDUM.

Cano, María Isabel y Lledó, Ángel. (1990). Espacio, comunicación y aprendizaje. Madrid: Díada.

Cárdenas Zuluaga, Claudia. (2012). La diversidad en la diversidad. Módulo Educación para la diversidad. Maestría Educación desde la Diversidad. Manizales: Universidad de Manizales.

Casassus, Juan. (2000). Problemas de la gestión educativa en América Latina. Chile: UNESCO.

Cere, (1993). Evaluar el contexto educativo, Documento de estudio. Vitoria Ministerio de Educación y Cultura. Gobierno Vasco.

Conferencia Internacional de Educación. (2008). 48 ${ }^{a}$ reunión, Ginebra

Conferencia Mundial sobre Educación para Todos. (1990). Jomtien, Tailandia.

Congreso de la República de Colombia. (1994). Ley General de Educación. Ley 115.

Duarte, Jakeline. (2005). "Violencia en la escuela. Comunicación y convivencia escolar en la ciudad de Medellín", en: Revista Iberoamericana de Educación (37).
Esteve, José Manuel, Franco, Soledad y Vera, Julio. (1995). Los profesores ante el cambio social. Barcelona: Anthropos.

Frasser, Nancy y Honneth, Axel. (2006)¿Redistribución o reconocimiento? Barcelona: Editorial Morata.

González, Fernando. (2008). Subjetividad social, sujeto y representaciones sociales. Bogotá Julio, vol.4 no.2 Diversitas.

González González, Miguel Alberto. (2011). Umbrales de indolencia. Educación sombría y justicia indiferente. Manizales: Universidad de Manizales.

González González, Miguel Alberto. (2009). Horizontes Humanos: Límites y paisajes. Manizales: Universidad de Manizales.

Isaza de Gil, Gloria. (2012). Aspectos Generales de la Políticas, Reformas y cambios Educativos. Módulo Grupos Vulnerables I. Maestría Educación desde la Diversidad. Manizales: Universidad de Manizales.

Mena, Isidora y Valdés, Ana María. (2008). Clima Social Escolar. Documento Valoras UC1. Santiago de Chile: Educar.

Krichesky, Marcelo. (2010). Sobre Inclusión Educativa y desafíos para el sistema educativo. $8^{\circ}$ Encuentro de la PEM.

Libreros, Daniel. (2002). Tensiones de las Políticas Educativas en Colombia. Balances y Perspectivas, Unidad de prospectivas y políticas educativas. Universidad Pedagógica Nacional de Colombia, Bogotá. 
Ministerio de Educación Nacional. (1994). Ley General de Educación. Colombia.

Ministerio de Educación Nacional (2005). Lineamientos de política para la atención educativa a poblaciones vulnerables. Bogotá: Magisterio

Ministerio de Educación Nacional. (2008). Serie de guías No 34. Guía para el mejoramiento institucional. De la autoevaluación al plan del mejoramiento. En: http://www.mineducacion.gov.co/1621/articles-177745_archivo_pdf.pdf (Recuperado en abril de 2012 )

Moscovici, Serge. (1979). La teoría de las representaciones Sociales, Athenea DigitalRevista de pensamiento e investigación social, 1(2). (350) Sevilla.

ONU. (1989). Convención sobre los Derechos del Niño. Tratado adoptado por la Asamblea General de las Naciones Unidas.

Patiño, Luz Elena. (2011). La atención a la diversidad en el contexto del aula de clase. Módulo Alternativas pedagógicas. Maestría Educación desde la Diversidad. Manizales: Universidad de Manizales.

Pérez, Teodoro. (2006). El Clima Escolar, Un Factor Clave En La Educación De Calidad. En: http://www.plandecenal.edu. co/html/1726/articles-308992_archivo.pdf. (Recuperado en abril de 2012).

Rodríguez, Nohelia. (2004). "El clima escolar". Revista digital, Investigación y educación, Número 7 , Volumen 3.

Sacristán, Gimeno. (1991). La gestión pedagógica de la escuela. (Gerencia educativa de la escuela)". Seminario Internacional de Gerencia Educativa, celebrado en México, 3 a 5 de junio, 1991.

Sepúlveda, John Alejandro. (2001). Borradores de la Psicología de la sensación y percepción. Armenia, Quindío, Facultad de Psicología, Universidad Antonio Nariño.

Soto Builes, Norelly. (2008). Representaciones Sociales y Discapacidad. Manizales: Facultad de Ciencias Sociales y Humanas: Universidad de Manizales.

Tagiuri, R. y Litwin, G. (1968). Organizational climate: Explorations of a concept. Boston: Harvard Business School.

UNESCO (1994). Declaración y marco de Acción sobre Necesidades Educativas Especiales. Conferencia Mundial sobre Ne- cesidades Educativas Especiales. Acceso y Calidad. Salamanca, España.

Zemelman, Hugo. (2008). Los retos de las ciencias sociales Latinoamericanas. Pensar teórico y pensar epistémico. Instituto de Pensamiento y Cultura en América Latina, IPECAL. México D.F.

\section{Referencias}

Arón, Ana María y Milicic, Neva. (1999). Clima social escolar y desarrollo personal. Un programa de mejoramiento. Santiago: Andrés Bello.

Arroyave, Santiago (2010). Políticas públicas en Colombia, insuficiencias y desafíos .Bogotá: Ediciones Aurora.

Gil, Inés. (2007). "Percepciones de la diversidad y de los logros escolares. Análisis desde un enfoque intercultural." Emigra. Madrid. Working papers, 87. En: www.emigra.org. es (Recuperado en agosto de 2012).

González González, Miguel Alberto. (2011). Resistir en la esperanza. Tertulias con el tiempo. Pereira: Universidad Tecnológica de Pereira.

González González, Miguel Alberto. (2012). Desafíos de la universidad. Miradas plurales. Carpe Diem. Madrid: Editorial Académica Española.

González González, Miguel Alberto. (2014). Miedos y olvidos pedagógicos. Rosario, Argentina: Editorial Homosapiens y UCP.

González González, Miguel Alberto. (2014). Metáforas y paradojas de los miedos en los sujetos docentes. Revista Latinoamericana de Ciencias Sociales, Niñez y Juventud, 12 (1), pp. 355-370.

Grisales Grisales, Carmenza. (2013). Seminario. Manizales: Universidad de Manizales.

Guarín Jurado, Germán. (2013). Seminario. Manizales: Universidad de Manizales

Hernández, Gregorio (2011). Paradigmas que fundamentan el concepto de cultura. Módulo Concepto de Cultura: Una Mirada SocioAntropológica Cultural. Maestría Educación desde la Diversidad. Manizales: Universidad de Manizales.

Isaza de Gil, Gloria. (2013). Seminario de investigación. Manizales: Universidad de Manizales.

Kliksberg, Bernardo. (1999). Desigualdad y desarrollo en América Latina: el debate 
postergado, en; Revista Reforma y Democracia (14).

Menjura, María Inés. (2013). Seminario de investigación. Manizales: Universidad de Manizales.

Moncayo, María. (2009). "El rol del maestro en los diferentes estilos de aprender" En: http:// www.planamanecer.com/recursos/docente/ bachillerato/articulos_pedagogicos/febrero/ diferencias_en_aprender.pdf

ONU. (1993). Sobre la igualdad de oportunidades para las personas con discapacidad. Cuadragésimo octavo período de sesiones,
Naciones Unidas, Consejo económico y social. Resolución 48/96, del 20 de diciembre de 1993.

Pozo, Juan. (2003). Adquisición dl conocimiento. Editorial Morata. Madrid.

Ramírez Aristizabal, Beatriz. (2013). Seminario presentación maestría. Manizales: Universidad de Manizales.

Soto, Norelly. (2008). Representaciones Sociales y Discapacidad. Manizales: Facultad de Ciencias Sociales y Humanas: Universidad de Manizales. 